\title{
Widespread extrahippocampal NAA/(Cr+Cho) abnormalities in TLE with and without mesial temporal sclerosis
}

\author{
Susanne G. Mueller • Andreas Ebel · Jerome Barakos • \\ Cathy Scanlon · Ian Cheong · Daniel Finlay · Paul Garcia • \\ Michael W. Weiner $\cdot$ Kenneth D. Laxer
}

Received: 26 July 2010/Revised: 29 September 2010/ Accepted: 7 October 2010/Published online: 26 October 2010

(C) The Author(s) 2010. This article is published with open access at Springerlink.com

\begin{abstract}
MR spectroscopy has demonstrated extrahippocampal $\mathrm{NAA} /(\mathrm{Cr}+\mathrm{Cho})$ reductions in medial temporal lobe epilepsy with (TLE-MTS) and without (TLE-no) mesial temporal sclerosis. Because of the limited brain coverage of those previous studies, it was, however, not possible to assess differences in the distribution and extent of these abnormalities between TLE-MTS and TLE-no. This study used a 3D whole brain echoplanar spectroscopic imaging (EPSI) sequence to address the following questions: (1) Do TLE-MTS and TLE-no differ regarding severity and distribution of extrahippocampal NAA/ $(\mathrm{Cr}+\mathrm{Cho})$ reductions? (2) Do extrahippocampal NAA/ $(\mathrm{Cr}+\mathrm{Cho})$ reductions provide additional information for focus lateralization? Forty-three subjects (12 TLE-MTS, 13 TLE-no, 18 controls) were studied with 3D EPSI. Statistical parametric mapping (SPM2) was used to identify regions of significantly decreased $\mathrm{NAA} /(\mathrm{Cr}+\mathrm{Cho})$ in TLE groups and in individual patients. TLE-MTS and TLE-no
\end{abstract}

S. G. Mueller · A. Ebel · C. Scanlon - I. Cheong · D. Finlay · M. W. Weiner

Center for Imaging of Neurodegenerative Diseases

and Department of Radiology and Biomedical Imaging,

University of California, San Francisco, CA, USA

J. Barakos · K. D. Laxer

Pacific Epilepsy Program, California Pacific Medical Center,

San Francisco, CA, USA

P. Garcia

Department of Neurology, University of California,

San Francisco, CA, USA

S. G. Mueller $(\square)$

Department of Veterans Affairs (DVA) Medical Center,

Center for Imaging of Neurodegenerative Diseases,

Clement Street 4150, San Francisco, CA 94121, USA

e-mail: susanne.mueller@ucsf.edu had widespread extrahippocampal $\mathrm{NAA} /(\mathrm{Cr}+\mathrm{Cho})$ reductions. $\mathrm{NAA} /(\mathrm{Cr}+\mathrm{Cho})$ reductions had a bilateral frontotemporal distribution in TLE-MTS and a more diffuse, less well defined distribution in TLE-no. Extrahippocampal $\mathrm{NAA} /(\mathrm{Cr}+\mathrm{Cho})$ decreases in the single subject analysis showed a large inter-individual variability and did not provide additional focus lateralizing information. Extrahippocampal NAA/(Cr+Cho) reductions in TLE-MTS and TLE-no are neither focal nor homogeneous. This reduces their value for focus lateralization and suggests a heterogeneous etiology of extrahippocampal spectroscopic metabolic abnormalities in TLE.

Keywords Temporal lobe epilepsy - Mesial temporal sclerosis $\cdot$ NAA $\cdot$ Spectroscopy

\section{Introduction}

Temporal lobe epilepsy (TLE) is the most common form of partial epilepsy. Two types of non-lesional medial TLE are distinguished based on imaging and histopathological findings: (1) TLE with mesial temporal lobe sclerosis (TLE-MTS, about 60-70\%), which is characterized by an atrophied hippocampus with MR signal abnormalities and severe neuronal loss in the histological examination, and (2) TLE with a normal appearing hippocampus on the MRI (TLE-no, about 30-40\%) and no or mild neuronal loss in the histological examination. The differences between the two TLE types, however, are not restricted to the hippocampus and its appearance in the MRI or histopathological preparation. Recent neuroimaging studies have shown that structural and functional abnormalities exist beyond the hippocampus and even beyond the temporal lobe, and that the two groups not only differ regarding severity of these 
abnormalities but more importantly also regarding their distribution [1-5]. These findings, together with other crucial clinical differences between TLE-MTS and TLEno, e.g. history of febrile seizures, age at onset of epilepsy, initial ictal zone [6] or success of epilepsy surgery [7, 8], suggest that TLE-no is more than just a milder form of TLE-MTS but eventually even a distinct entity of TLE.

MR spectroscopy allows the non-invasive measurement of $\mathrm{N}$-acteyl-aspartate (NAA) (marker of neuronal viability and functionality), creatine/phosphocreatine (Cr) (marker of energy metabolism), and choline compounds (Cho) (marker of cell membrane integrity), simultaneously and thus provides information about various important aspects of the brain metabolism. The typical spectroscopic hallmark of the epileptogenic focus is an NAA reduction which is generally thought to represent mostly neuron loss $[9,10]$, while Cho and $\mathrm{Cr}$ are unchanged [11]. Using multiple single voxel spectroscopy or multi-slice spectroscopic imaging techniques, it has been shown that NAA reductions are not restricted to the focus in TLE but can be found in extrahippocampal and extratemporal regions as well $[12,13]$. Because of their limited brain coverage, these previous studies were not able to conclusively assess if the distribution and/or extent of these extrafocal NAA reductions differs between TLE-MTS and TLE-no. The recent development of 3D spectroscopic imaging sequences, which provide spectroscopic information from the whole brain, has changed this [14]. Using such a sequence [15] we addressed the following specific aims: (1) Confirm the findings of previous studies showing that extrahippocampal/extratemporal NAA reductions exist in unilateral TLE and to test if TLE-MTS and TLE-no differ regarding the distribution and severity of these abnormalities. Based on recent findings in structural MRI [16], it was assumed that NAA reductions in TLE-MTS would show a mesial-temporal, temporalposterior to occipital distribution and in TLE-no a temporolateral distribution. (2) Test if severity and extent of extrahippocampal NAA reductions provide additional information for focus lateralization in TLE-MTS or TLE-no. It was assumed that they would be more prominent in the hemisphere and/or lobe containing the epileptogenic focus.

\section{Methods}

\section{Study population}

The committees of human research at the University of California, San Francisco (UCSF), California Pacific Medical Center, San Francisco (CPMC) and VA Medical Center, San Francisco approved the study, and written informed consent was obtained from each subject according to the Declaration of Helsinki. Twenty-five consecutive patients suffering from therapy resistant temporal lobe epilepsy with unilateral seizure onset who were evaluated for epilepsy surgery and agreed to undergo the additional special research imaging and spectroscopy protocol at $4 \mathrm{~T}$ were recruited between mid 2005 and the end of 2009 from the Pacific Epilepsy Program, CPMC and the Northern California Comprehensive Epilepsy Center, UCSF. Twelve (mean age $39.6 \pm 11.5$; left TLE/right TLE: 7/5, females/ males 8/4) had evidence for mesial temporal lobe sclerosis (MTS) on their MRIs (TLE-MTS) and the remaining 13 (mean age $40.5 \pm 9.5$; left TLE/right TLE: $8 / 5$; females/ males: 7/6) had normal appearing hippocampi (TLE-no). The findings regarding presence/absence of MTS were confirmed using subfield volumetry in high resolution T2 weighted images [17]. The two groups differed regarding mean age at onset of epilepsy (TLE-MTS: $11.2 \pm 10.0$ years; TLE-no: $26.2 \pm 12.4$ years, $p=0.003$ ) and mean duration of epilepsy (TLE-MTS: $28.9 \pm 15.6$ years; TLEno: $14.8 \pm 12.4$ years; $p=0.02$ ). The identification of the epileptogenic focus was based on seizure semiology and prolonged ictal and interictal Video/EEG/Telemetry (VET) in all patients. In order to qualify as unilateral TLE, all seizures (average $4.8 \pm 2.4$ /patient) recorded during this time had to be typical for the patient, show EEG features consistent with mesial temporal onset and arise from the same side. All TLE subjects reported having been seizure free for at least $24 \mathrm{~h}$ before the $4 \mathrm{~T}$ study. The seizure frequency was determined by calculating the mean frequency/ month of all seizure types recorded by the patient in the three months before the research MR exam. Table 1 displays the patient characteristics. The control population consisted of 18 healthy age-matched volunteers (mean age $33.5 \pm 10.0$; females/males: 13/5).

\section{MRI acquisition}

All studies were performed on a Bruker MedSpec 4T system controlled by a Siemens Trio ${ }^{\mathrm{TM}}$ console and equipped with a USA instruments eight channel array coil. The following sequences, which were part of a larger research imaging and spectroscopy protocol, were acquired: (1) volumetric T1weighted gradient echo MRI (MPRAGE) TR/TE/ $\mathrm{TI}=2,300 / 3 / 950 \mathrm{~ms}, 7^{\circ}$ flip angle, $1.0 \times 1.0 \times 1.0 \mathrm{~mm}^{3}$ resolution, acquisition time $5 \mathrm{~min}$. (2) 3D echo planar whole brain spectroscopic imaging (EPSI) TR/TE/TI $=1,780$ / $45 / 280 \mathrm{~ms}, \mathrm{FOV}=280 \times 280 \times 180 \mathrm{~mm}^{3}(x, y, z)$, spatial matrix: $50 \times 50 \times 18$ points, spectral bandwidth $=$ $1,667 \mathrm{~Hz}, 800$ spectral points. Metabolite and water reference data was acquired in an interleaved fashion with the delay between metabolite and water reference excitations of $\mathrm{TR}_{\mathrm{WR}}=550 \mathrm{~ms}$. Total EPSI acquisition time: $27 \mathrm{~min}$. This spectroscopic imaging sequence allows for the detection of the major brain metabolites NAA, $\mathrm{Cr}$ and Cho. 
Table 1 Patient characteristics

\begin{tabular}{|c|c|c|c|c|c|c|c|}
\hline Pat no. & Age/sex & $4 \mathrm{~T}$ MRI & Focus & Onset & Duration & Risk factors & Histo/outcome \\
\hline 1 & $35 / \mathrm{m}$ & L MTS L temp atrophy & L mesial temp & 4 & 31 & & L temp/MTS/I/24 \\
\hline 2 & $50 / f$ & L MTS L temp atrophy & L mesial temp & 2 & 49 & FS & $\mathrm{L}$ temp/MTS/I/6 \\
\hline 3 & $35 / f$ & L MTS & L mesial temp & 9 & 26 & & L temp/MTS/II/12 \\
\hline 4 & $38 / f$ & L MTS & L mesial temp & 6 & 32 & FS & na \\
\hline 5 & $53 / \mathrm{f}$ & L MTS & $\mathrm{L}$ mesial temp & 28 & 26 & Infection & L temp/MTS/I/6 \\
\hline 6 & $32 / f$ & L MTS & L mesial temp & 24 & 9 & Infection & L temp/MTS/IV/6 \\
\hline 7 & $49 / f$ & R MTS & $\mathrm{R}$ mesial temp & 7 & 42 & & R temp/MTS/I/24 \\
\hline 8 & $23 / \mathrm{f}$ & R MTS & $\mathrm{R}$ mesial temp & 5 & 18 & Infection & na \\
\hline 9 & $33 / \mathrm{f}$ & L MTS & L mesial temp & 14 & 20 & & na \\
\hline 10 & $63 / \mathrm{m}$ & R MTS & $\mathrm{R}$ mesial temp & 6 & 58 & FS & na \\
\hline 11 & $32 / \mathrm{m}$ & R MTS & $\mathrm{R}$ mesial temp & 28 & 4 & & $\mathrm{R}$ temp/MTS/I/9 \\
\hline 12 & $32 / \mathrm{m}$ & R MTS, subtle WMH & $\mathrm{R}$ mesial temp & 1 & 32 & Infection & $\mathrm{R}$ temp/MTS/I/4 \\
\hline 13 & $39 / \mathrm{m}$ & Small cyst L temp pole & L mesial temp & 29 & 11 & & na \\
\hline 14 & $27 / f$ & Normal & L mesial temp & 10 & 17 & & na \\
\hline 15 & $33 / f$ & $\mathrm{~L}$ front $\mathrm{WMH}$ & L mesial temp & 10 & 24 & & L temp/MTS/I/18 \\
\hline 16 & $38 / \mathrm{f}$ & Normal & L mesial temp & 37 & 2 & & na \\
\hline 17 & $22 / f$ & Normal & $\mathrm{R}$ mesial temp & 22 & 0 & & na \\
\hline 18 & $45 / \mathrm{f}$ & Normal & $\mathrm{R}$ mesial temp & 31 & 15 & & na \\
\hline 19 & $45 / \mathrm{m}$ & WMH & $\mathrm{R}$ mesial temp & 30 & 16 & Posttrauma & R temp/norm/I/18 \\
\hline 20 & $41 / \mathrm{m}$ & Normal & $\mathrm{R}$ mesial temp & 40 & 1 & Posttrauma & na \\
\hline 21 & $45 / \mathrm{m}$ & Normal & $\mathrm{R}$ mesial temp & 38 & 8 & & na \\
\hline 22 & $57 / \mathrm{m}$ & Normal & $\mathrm{L}$ mesial temp & 13 & 44 & & na \\
\hline 23 & $53 / \mathrm{f}$ & Normal & L mesial temp & 47 & 6 & & na \\
\hline 24 & $42 / \mathrm{f}$ & Normal & L mesial temp & 21 & 21 & & na \\
\hline 25 & $39 / \mathrm{m}$ & Normal & L mesial temp & 13 & 27 & & L temp/norm/I/24 \\
\hline
\end{tabular}

Age/sex age at examination/sex, $f$ female, $m$ male, MTS mesial temporal sclerosis at $1.5 \mathrm{~T}$, Norm normal MRI at $1.5 \mathrm{~T}, R$ right, $L$ left, temp temporal, WMH white matter hyperintensities, Onset age at onset, Duration duration of epilepsy, FS febrile seizure, Infection history of encephalitis/meningitis without temporal relationship to onset of seizures, perinatal perinatal complications, posttrauma mild closed head trauma in history without temporal relationship to onset of seizures, histo histology, surgery epilepsy surgery, na no. surgery, Outcome latest outcome using Engel's classification is given in roman numerals, follow-up in months is given in arabic numerals

Postprocessing

\section{EPSI postprocessing}

A B0 map was derived from the water reference image. The water reference data was then zero-filled from $50 \times 50 \times 18$ to $64 \times 64 \times 24$ points, and a spatial apodization using a Gaussian filter was applied before performing of a four-dimensional spectral FT and B0 correction [18]. The resulting image was used to generate a brain mask which was applied to the metabolite data to avoid fitting of regions of no interest. The metabolite data was corrected for chemical shift artifact, echo averaged and the residual water signal was reduced by applying a finite impulse response (FIR) filter. Spectral processing of the metabolite data included zero filling to 512 points, lipid $k$ space extrapolation and Gaussian apodization with a line broadening of $2 \mathrm{~Hz}$ before performing a four-dimensional
FT and spectral fitting with SITOOLS [19] (cf. Fig. 1). A binary map containing only voxels of acceptable quality (QC map) was generated using linewidth (between 3-21.3 Hz) and fit accuracy (fits with residual sum squares outside the 95 percentile distribution of residuals from all fits were rejected) $[18,20]$ as main criteria. Intensity images for each metabolite of interest (NAA, Cr and Cho) were derived from the fitted metabolite data and used to calculate $\mathrm{NAA} /(\mathrm{Cr}+\mathrm{Cho})$ maps. $\mathrm{NAA} /(\mathrm{Cr}+\mathrm{Cho})$ has been found to be very sensitive to the type of metabolic abnormality observed in TLE [13] and has (compared to single metabolite maps) the additional advantage that it does not require an intensity non uniformity correction of the spectral data. Voxels with $\mathrm{NAA} /(\mathrm{Cr}+\mathrm{Cho})>4$ indicating an insufficient suppression/extraction of the skull lipid signal were excluded. The final QC map was applied to the resulting ratio map to eliminate spectra with insufficient quality. 


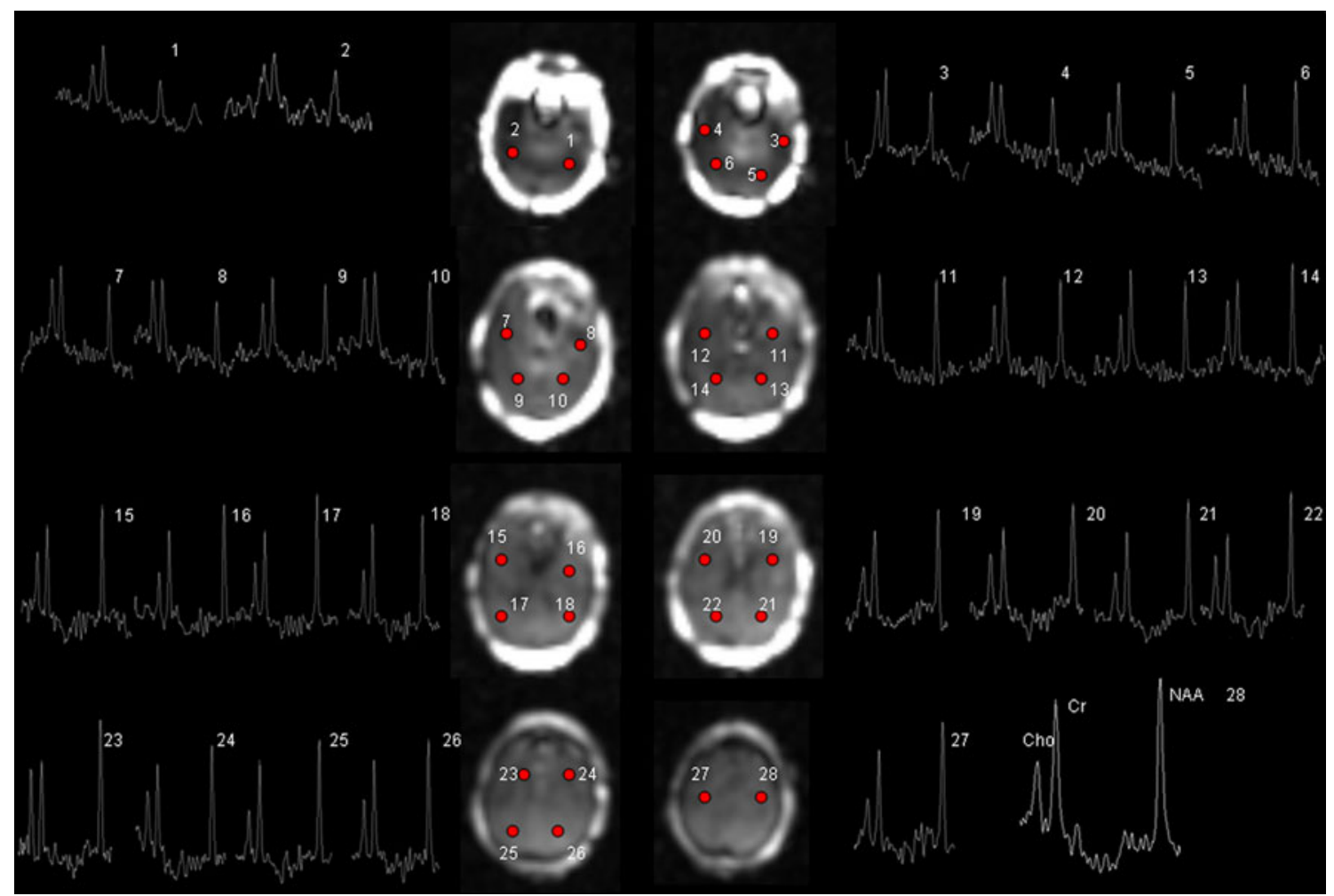

Fig. 1 Raw 3D EPSI with representative spectra from a TLE-MTS patient with a right sided focus. All displayed spectra passed the automated quality control. Spectrum 28 has been enlarged and the

\section{Structural image processing}

All T1 images were segmented in gray, white and CSF tissue maps using expectation maximization segmentation (EMS) [21, 22].

\section{Combination of structural and EPSI data}

The T1 images were co-registered (six affine, mutual information [23]), down-sampled to the resolution of the EPSI water reference image and the resulting transformation matrices were applied to the $\mathrm{T} 1$ image and the gray matter map. The resulting down-sampled gray matter maps were spatially normalized to a previously generated customized symmetrical gray matter prior in ICBM (International Consortium for Brain Mapping) space using the spatial normalization algorithm in statistical parametric mapping 2 (SPM2) (Wellcome Department of Cognitive Neurology, http://www.fil.ion.ucl.ac.uk) running MATLAB 6.1 (The MathWorks, Natick, MA, USA). The resulting transformations were then applied to the NAA/ $(\mathrm{Cr}+\mathrm{Cho})$ map with and without QC, the QC map, the T1 image co-registered to the EPSI and the gray matter map. The resulting spatially normalized maps were re-sampled to a $1 \times 1 \times 1 \mathrm{~mm}$ resolution. peaks of the main metabolites are labelled. NAA N-acetyl-aspartate, $\mathrm{Cr}$ creatine/phosphocreatine, Cho choline compounds

\section{Data analysis}

Two approaches were used for data analysis: (1) Group analysis: each TLE subgroup was compared to the control group and with each other to identify regions with decreased $\mathrm{NAA} /(\mathrm{Cr}+\mathrm{Cho})$. In order to allow for the combination of left and right onset TLE patients, the NAA/ $(\mathrm{Cr}+\mathrm{Cho})$ maps of all right TLE were side flipped so that the hemisphere with the epileptogenic focus was on the left side in all patients. To account for physiological right/left hemispheric differences, all control NAA/(Cr+Cho) maps were also side-flipped and both versions were used for the group comparisons. (2) Single subject analysis: each individual TLE subject was compared to the control group and a map with regions of significantly reduced NAA/ $(\mathrm{Cr}+\mathrm{Cho})$ generated using SPM2.

Statistical analysis

\section{Group analysis}

SPM2 was used to identify regions with significantly decreased $\mathrm{NAA} /(\mathrm{Cr}+\mathrm{Cho})$ in TLE-MTS compared to controls, in TLE-no compared to controls, and in TLEMTS compared to TLE-no (compare populations:1 scan/ 
subject (ANCOVA), age as nuisance variable, threshold for significance at voxel level $p<0.001$ and $p<0.05$ corrected at cluster level).

\section{Single subject analysis}

SPM2 was used to localize regions of metabolic abnormalities in individual TLE subjects by performing a $t$ test in which each TLE subject was compared with all control subjects [compare populations: 1 scan/subject (two-sample $t$ test)]. The threshold for statistical significance was defined as $p<0.005$ at voxel level and $p<0.05$ at cluster level. These thresholds had been determined empirically by comparing each control with all remaining controls and then choosing the threshold at which none of the controls except one had clusters with abnormally low NAA/ $(\mathrm{Cr}+\mathrm{Cho})$. In this control, these clusters persisted even when higher thresholds (FDR $p<0.05$ ) were applied. The localization of each cluster was based on the coordinates of the voxel with the maximal $t$ value. The region with the largest cluster of significantly reduced NAA/ $(\mathrm{Cr}+\mathrm{Cho})$ was arbitrarily determined to represent the "focus as determined by 3D EPSI". If the "focus as determined by 3D EPSI" was concordant with the epileptogenic temporal lobe as identified by VET, the EPSI focus identification was considered to be "correct". EPSI identification of the epileptogenic temporal lobe instead of the hippocampus was chosen because the spectral quality in the hippocampus was often adversely affected by the susceptibility artifacts typical for the basal temporal lobe. This resulted in the loss of the spectra from the head and anterior two-thirds of the body of the hippocampus (on average $26 \pm 14 \%$ of the hippocampal voxels fulfilled the quality criteria), i.e., the region where previous studies had shown the most prominent abnormalities in TLE [11]. Because of this, spectral information from the hippocampal tail was lumped together with the temporal lobe for "focus determination by EPSI". Mann-Whitney tests and Fisher's exact test were used to compare indices of epilepsy severity and EPSI brain coverage between TLE with and without clusters of abnormally low NAA/(Cr+Cho). Holms' test was used to correct for multiple comparisons.

\section{Results}

Group analysis

Figure 2 displays the results of the group analysis. NAA/ $(\mathrm{Cr}+\mathrm{Cho})$ abnormalities in TLE-MTS had a bilateral temporo-occipital distribution. Additional prominent abnormalities were present in both frontal lobes. NAA/ $(\mathrm{Cr}+\mathrm{Cho})$ abnormalities in TLE-no were more diffuse and less well-defined and were most prominent in the temporoinsular and bilateral frontal regions. The direct comparisons between TLE-MTS and TLE-no showed no significant differences at the chosen significance threshold.

Single subject analysis

Figure 3 displays the results of the single subject analysis for two typical TLE-MTS and two typical TLE-no. Table 2 shows the results for each subject. Three TLE-MTS and five TLE-no had no clusters with significant NAA/ $(\mathrm{Cr}+\mathrm{Cho})$ reductions. The remaining nine TLE-MTS had 1-5 clusters with abnormally low $\mathrm{NAA} /(\mathrm{Cr}+\mathrm{Cho})$ [average number (No) of clusters: 2.7; median cluster size: 93,021 (range 1,193-602,663 voxels; median size of largest cluster: 4,515 (range 1,193-5,85,555 voxels)], and the remaining eight TLE-no had 1-7 clusters with abnormally low NAA/(Cr+Cho) [average No of clusters: 4.0; median cluster size: 11,287 (range 1,638-49,841) voxels; median size of largest cluster: 4,828 (range 1,638-26,961 voxels)]. TLE-MTS and TLE-no did not differ from each other regarding number of abnormal $\mathrm{NAA} /(\mathrm{Cr}+\mathrm{Cho})$ clusters nor regarding the size of these clusters or size of the largest cluster.

The largest cluster of abnormally low NAA/ $(\mathrm{Cr}+\mathrm{Cho})$ was in the ipsilateral temporal lobe in five TLE-MTS. In the remaining four TLE-MTS, the largest cluster with abnormally low $\mathrm{NAA} /(\mathrm{Cr}+\mathrm{Cho})$ was either in the contralateral temporal lobe $(n=3)$ or contralateral $(n=1)$ frontal lobe. In seven TLE-no the largest cluster with abnormally low $\mathrm{NAA} /(\mathrm{Cr}+\mathrm{Cho})$ was found in the contralateral hemisphere (frontal: 3 ; insula: 2; parietal: 1, temporal: 1) and in one it was found in the ipsilateral occipital lobe.

Temporal lobe epilepsy with abnormally low NAA/ $(\mathrm{Cr}+\mathrm{Cho})$ did not differ from TLE with $\mathrm{NAA} /(\mathrm{Cr}+\mathrm{Cho})$ within the normal range regarding mean duration of epilepsy (22.3 \pm 17.5 vs. $20.3 \pm 10.9$ years, $p=0.74)$, age at onset of epilepsy (19.5 \pm 13.8 vs. $18.0 \pm 13.5)$ occurrence of secondary generalized seizures (23.5 vs. $12.5 \%$, $p=0.47$ ), occurrence of risk factors for epilepsy (35.3 vs. $37.5 \%, p=0.71)$, seizures/month $(7.5 \pm 15.3$ vs. $11.9 \pm$ $14.8, p=0.52$ ) or $\%$ brain coverage by 3DEPSI (35.1 \pm 8.2 vs. $39.3 \pm 4.3 \%, p=0.19$ ).

\section{Discussion}

There were three major findings in this study: (1) Ipsi- and contralateral temporal and extratemporal regions with abnormally low NAA/ $(\mathrm{Cr}+\mathrm{Cho})$ were found in TLE-MTS and TLE-no. The extent of these $\mathrm{NAA} /(\mathrm{Cr}+\mathrm{Cho})$ reductions varied between subjects even within the same 
Fig. 2 Regions with significant decreased $\mathrm{NAA} /(\mathrm{Cr}+\mathrm{Cho})$ in TLE-MTS compared to controls (upper two panels) and TLE-no compared to controls (lower two panels). Abnormalities in TLEMTS were most prominent in the bilateral temporal lobes but extended also into posterior occipital and frontal regions. Abnormalities in TLE-no were more diffuse, less distinct and preferentially in bilateral frontal, temporal-insular regions. Similarly, as in the single subject analysis, there was no clear predominance of the $\mathrm{NAA} /(\mathrm{Cr}+\mathrm{Cho})$ reductions in the ipsilateral hemisphere in either group

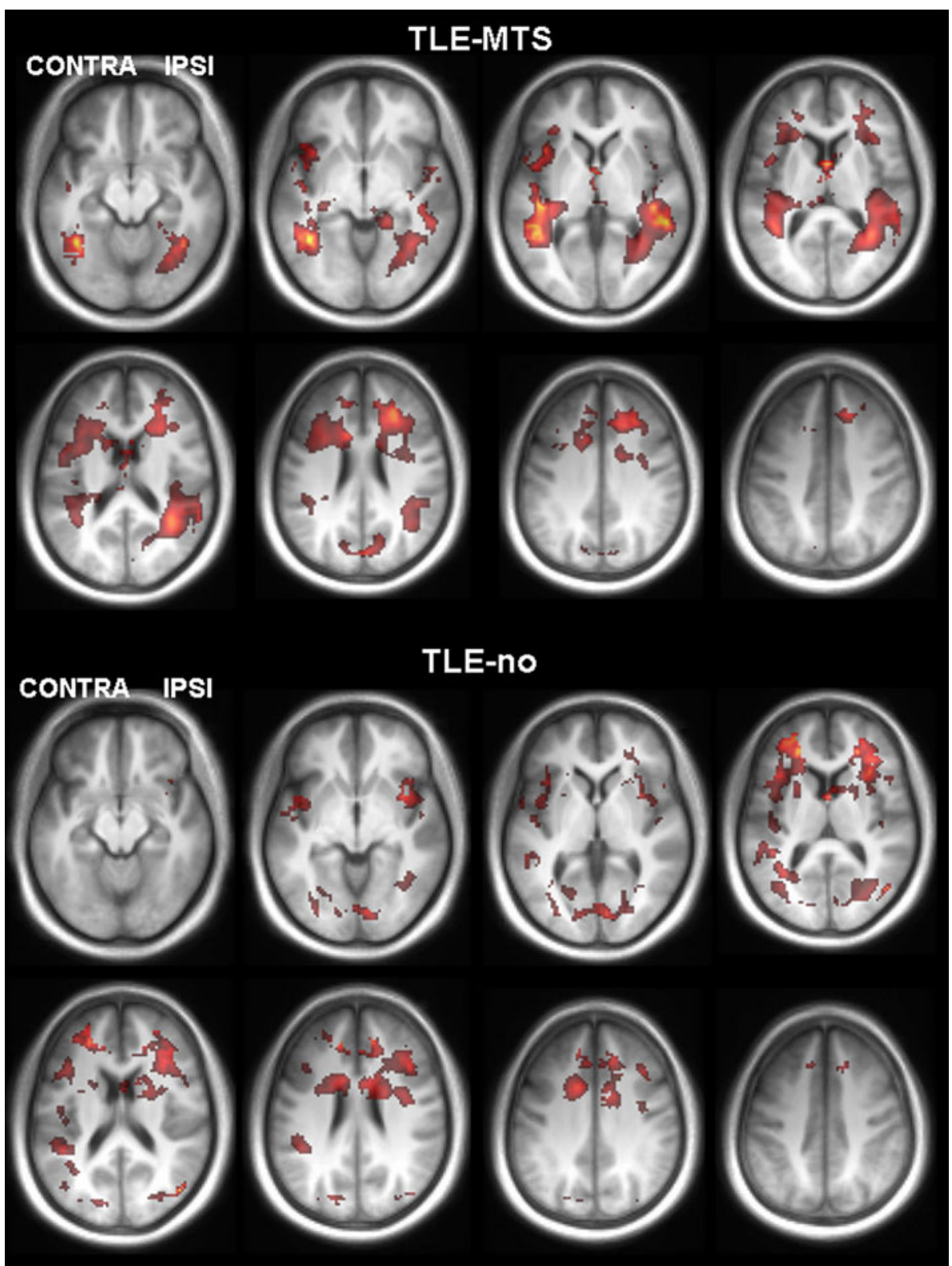

subgroup. Factors that might influence the expression of these abnormalities, e.g., seizure frequency, type, were not different between TLE with and without widespread NAA/ $(\mathrm{Cr}+\mathrm{Cho})$ reductions. (2) The high degree of interindividual heterogeneity and variability of the metabolic abnormalities complicated the identification of a distinct pattern of NAA/ $(\mathrm{Cr}+\mathrm{Cho})$ reductions in each of the two TLE subgroups. Although the visual inspection of the TLE vs. control comparisons suggested a predominantly temporal-frontal distribution of the $\mathrm{NAA} /(\mathrm{Cr}+\mathrm{Cho})$ decreases in TLE-MTS and a more diffuse, less distinct distribution in TLE-no (cf. Fig. 2), the two TLE groups were not significantly different from each other in the direct comparison. (3) Extrafocal NAA/(Cr+Cho) abnormalities did not provide additional information for focus lateralization in
TLE-MTS or in TLE-no. Taken together, this study provides evidence that at least metabolically non-lesional TLE is neither a focal nor a homogeneous disease.

\section{Etiology of extrafocal $\mathrm{NAA} /(\mathrm{Cr}+\mathrm{Cho})$ reductions}

The first finding of this study was the confirmation of the widespread, bilateral extrafocal $\mathrm{NAA} /(\mathrm{Cr}+\mathrm{Cho})$ reductions in TLE-MTS and TLE-no which had been described by previous spectroscopic imaging studies with partial brain coverage $[12,13]$. The etiology of these extrahippocampal $\mathrm{NAA} /(\mathrm{Cr}+\mathrm{Cho})$ reductions in TLE is unknown. NAA decreases in the hippocampus are usually thought to be caused by neuron loss and/or neuronal dysfunction [9]. The fact that hippocampal NAA decreases have been found to 
Fig. 3 Representative results of the single subject analysis. The two TLE-MTS had large ipsilateral temporal clusters which extended in neighboring regions. There were also smaller clusters in the frontal lobes. The two TLE-no in general had smaller and more diffusely distributed clusters

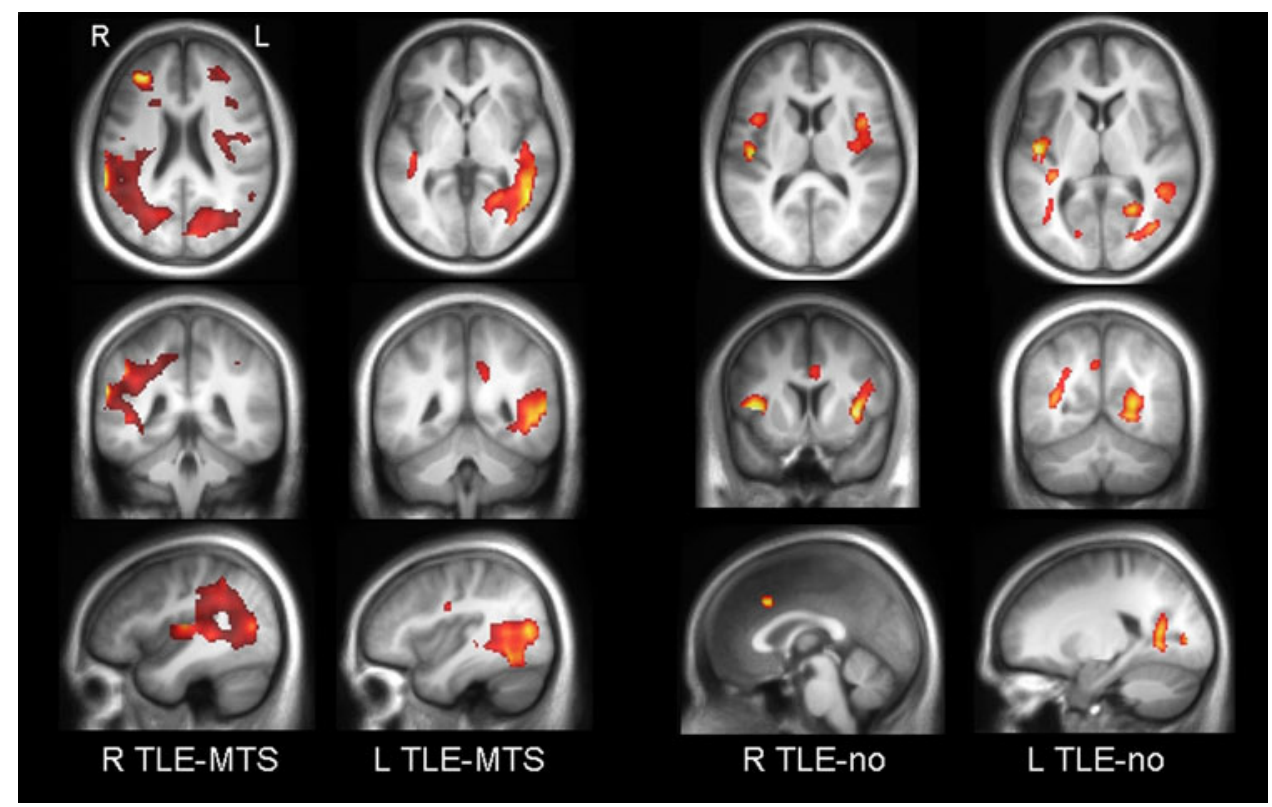

be useful for focus lateralization in TLE [24] suggests that they are stable over time, at least in the epileptogenic hippocampus. Neuronal loss and neuronal dysfunction could also explain the extrahippocampal and extratemporal $\mathrm{NAA} /(\mathrm{Cr}+\mathrm{Cho})$ reductions found in this study. In analogy to the focus, local excitotoxic effects of spreading epileptogenic activity could lead to neuronal loss in extrafocal regions. In that case it could be expected that the resulting $\mathrm{NAA} /(\mathrm{Cr}+\mathrm{Cho})$ abnormalities are associated with structural abnormalities/volume loss. Widespread cortical thinning in TLE-MTS and TLE-no with a similar distribution as the $\mathrm{NAA} /(\mathrm{Cr}+\mathrm{Cho})$ reductions described here has indeed recently been demonstrated in a study from this lab [16]. Extrafocal neuronal dysfunction could be caused by deafferentation due to loss of input from the focus, or by excitotoxic effects of seizure spread too mild to result in neuronal loss. The best evidence that $\mathrm{NAA} /(\mathrm{Cr}+\mathrm{Cho})$ reductions due to neuronal dysfunction exist in TLE comes from the observation that reduced NAA in the contralateral hippocampus often returns to normal levels after successful surgery $[25,26]$. However, the observation that some of the extrafocal $\mathrm{NAA} /(\mathrm{Cr}+\mathrm{Cho})$ abnormalities are reversible also indicates that the severity and extent of these NAA/ $(\mathrm{Cr}+\mathrm{Cho})$ reductions are not necessarily stable over time. Longitudinal studies correlating extrahippocampal/extratemporal $\mathrm{NAA} /(\mathrm{Cr}+\mathrm{Cho})$ changes with structural abnormalities and clinical variables, such as seizure frequency between exams, will be necessary to explore in which regions extrafocal $\mathrm{NAA} / \mathrm{Cr}+\mathrm{Cho})$ reductions are stable over time and correspond to an irreversible neuron loss, and in which regions they fluctuate and indicate neuronal dysfunction.
High intersubject variability of $\mathrm{NAA} /(\mathrm{Cr}+\mathrm{Cho})$ reductions in TLE-MTS and TLE-no

The single subject analysis showed a wide range of these extrafocal $\mathrm{NAA} /(\mathrm{Cr}+\mathrm{Cho})$ reductions even within the same TLE group with some being metabolically completely normal while others had severe, widespread abnormalities. The high degree of intersubject variability of $\mathrm{NAA} /(\mathrm{Cr}+\mathrm{Cho})$ abnormalities and the relatively small population sizes made it difficult to identify a distinct pattern of metabolic abnormalities in each of the TLEsubgroups (cf. Fig. 2). The most obvious explanation for the variability of $\mathrm{NAA} /(\mathrm{Cr}+\mathrm{Cho})$ reductions between patients would be differences in severity and duration of the epileptic disorder. Several studies have described a relationship between structural abnormalities and duration of epilepsy [27, 28]. Therefore, it could be expected that TLE patients with a longer duration of epilepsy, more frequent and/or more severe seizures (occurrence of secondary generalized seizures) have more severe NAA/ $(\mathrm{Cr}+\mathrm{Cho})$ reductions than subjects with less frequent and/ or milder seizures or who developed seizures later in life. However, the direct comparison of metabolically normal TLE with metabolically abnormal TLE provided no evidence that any of these factors had an influence on the severity of the $\mathrm{NAA} /(\mathrm{Cr}+\mathrm{Cho})$ reductions. Technical issues which could have caused such variations artificially, i.e., differences in \% brain coverage between metabolically normal and abnormal subjects resulting in the exclusion of regions with reduced $\mathrm{NAA} / \mathrm{Cr}+\mathrm{Cho})$ in the former but inclusion in the latter, also seem unlikely since \% brain coverage was not different between subjects with normal 
Table 2 Results of single subject analysis

\begin{tabular}{|c|c|c|c|c|c|}
\hline Pat no. & Group & No. cluster & Size cluster & Side & Lobe \\
\hline 1 & MTS & 0 & 0 & na & na \\
\hline \multirow[t]{2}{*}{2} & MTS & 2 & 4,515 & Contra & Frontal \\
\hline & & & 2,081 & IPSI & Temporal \\
\hline \multirow[t]{5}{*}{3} & MTS & 5 & 35,365 & IPSI & Temporal \\
\hline & & & 2,258 & Contra & Temporal \\
\hline & & & 1,408 & IPSI & Frontal \\
\hline & & & 2,342 & Contra & Frontal \\
\hline & & & 1,420 & IPSI & Parietal \\
\hline 4 & MTS & 1 & 1,193 & Contra & Temporal \\
\hline 5 & MTS & 1 & 1,652 & Contra & Temporal \\
\hline 6 & MTS & 0 & 0 & na & na \\
\hline \multirow[t]{5}{*}{7} & MTS & 5 & 9,708 & IPSI & Frontal \\
\hline & & & 97,853 & IPSI & Temporal \\
\hline & & & 12,774 & Contra & Frontal \\
\hline & & & 1,784 & Contra & Temporal \\
\hline & & & 5,487 & Contra & Frontal \\
\hline 8 & MTS & 1 & 1,295 & IPSI & Temporal \\
\hline 9 & MTS & 1 & 2,481 & Contra & Temporal \\
\hline \multirow[t]{4}{*}{10} & MTS & 4 & 15,170 & Contra & Frontal \\
\hline & & & 21,965 & IPSI & Temporal \\
\hline & & & 3,773 & Contra & Temporal \\
\hline & & & 10,002 & Contra & Parietal \\
\hline \multirow[t]{4}{*}{11} & MTS & 4 & 6,609 & IPSI & Frontal \\
\hline & & & 1,350 & Contra & Frontal \\
\hline & & & 585,555 & IPSI & Temporal \\
\hline & & & 9,149 & Contra & Temporal \\
\hline 12 & MTS & 0 & 0 & na & na \\
\hline \multirow[t]{7}{*}{13} & Norm & 7 & 2,966 & Contra & Insula \\
\hline & & & 1,147 & Contra & Temporal \\
\hline & & & 1,303 & IPSI & Frontal \\
\hline & & & 7,799 & Contra & Parietal \\
\hline & & & 6,211 & IPSI & Occipital \\
\hline & & & 1,326 & Contra & Frontal \\
\hline & & & 2,239 & IPSI & Temporal \\
\hline 14 & Norm & 1 & 1,638 & Contra & Insula \\
\hline 15 & Norm & 0 & 0 & na & na \\
\hline \multirow[t]{5}{*}{16} & Norm & 5 & 1,740 & Contra & Occipital \\
\hline & & & 1,792 & Contra & Frontal \\
\hline & & & 1,269 & IPSI & Frontal \\
\hline & & & 1,257 & Contra & Temporal \\
\hline & & & 1,587 & IPSI & Occipital \\
\hline \multirow[t]{4}{*}{17} & Norm & 4 & 6,625 & Contra & Insula \\
\hline & & & 2,062 & IPSI & Insula \\
\hline & & & 2,625 & IPSI & Frontal \\
\hline & & & 3,121 & $\mathrm{Bi}$ & Frontal \\
\hline 18 & Norm & 0 & 0 & na & $\mathrm{Na}$ \\
\hline
\end{tabular}

Table 2 continued

\begin{tabular}{lllrll}
\hline Pat no. & Group & No. cluster & Size cluster & Side & Lobe \\
\hline 19 & Norm & 3 & 2,518 & IPSI & Frontal \\
& & & 2,708 & Contra & Parietal \\
& & & 2,915 & Contra & Frontal \\
20 & Norm & 0 & 0 & na & Na \\
21 & Norm & 4 & 2,532 & IPSI & Frontal \\
& & & 5,813 & Contra & Occipital \\
& & & 7,243 & Contra & Frontal \\
& & & 1,365 & IPSI & Temporal \\
22 & Norm & 1 & 3,030 & IPSI & Occipital \\
23 & Norm & 7 & 2,400 & Contra & Frontal \\
& & & 18,384 & IPSI & Occipital \\
& & & 3,983 & Contra & Frontal \\
& & & 18,552 & Contra & Temporal \\
& & & 3,102 & Bi & Frontal \\
& & & 2,228 & Bi & Frontal \\
& & & 1,192 & IPSI & Frontal \\
& & & 0 & na & na \\
& & & 0 & na & na \\
\hline
\end{tabular}

IPS ipsilateral, Contra contralateral, Bi bilateral, MTS mesial temporal sclerosis, Norm normal, No. cluster number of clusters, Size cluster number of voxels with abnormally low $\mathrm{NAA} /(\mathrm{Cr}+\mathrm{Cho})$ in the cluster, bold cluster which fulfill criterion for "focus as identified by EPSI" and are concordant with EEG identification of the focus

EPSI and those with abnormal EPSI. There are, of course, a multitude of other potential factors which alone or in combination might explain the wide range of NAA/ $(\mathrm{Cr}+\mathrm{Cho})$ abnormalities observed in non-lesional TLE, e.g., interval between last seizure and scan [29], type of the last seizure [30], type of antiepileptic medication, different individual vulnerability for effects of epileptogenic activity in non epileptogenic brain tissue, etc. Longitudinal studies in a larger patient population might help to identify the factors contributing to this variability.

Value of extrafocal $\mathrm{NAA} /(\mathrm{Cr}+\mathrm{Cho})$ reductions for focus lateralization

The lateralizing information provided by $\mathrm{NAA} /(\mathrm{Cr}+\mathrm{Cho})$ reductions in individual TLE-MTS patients was minimal. Only $42 \%$ of the TLE-MTS had the largest cluster of reduced $\mathrm{NAA} /(\mathrm{Cr}+\mathrm{Cho})$ in the ipsilateral temporal lobe and the hemispheric $\mathrm{NAA} /(\mathrm{Cr}+\mathrm{Cho})$ reductions were not significantly different between the ipsi- and contralateral hemispheres. The lateralizing information was even worse for TLE-no. There were no significant ipsi-/contralateral hemispheric differences and the largest cluster of low 
$\mathrm{NAA} /(\mathrm{Cr}+\mathrm{Cho})$ were in all cases extratemporal. Based on this, it can be concluded that extrahippocampal/extratemporal $\mathrm{NAA} /(\mathrm{Cr}+\mathrm{Cho})$ reductions do not provide additional information for focus lateralization. Further study will be needed to address the question if the extent and distribution of these extrafocal $\mathrm{NAA} /(\mathrm{Cr}+\mathrm{Cho})$ reductions might be useful to predict post-surgical seizure control or cognitive impairment.

\section{Technical limitations}

The study has limitations: (1) Loss of spectral data affected particularly the basal and mesial temporal lobe, i.e., regions which are prominently affected in TLE. Therefore, we may have missed significant temporal $\mathrm{NAA} /(\mathrm{Cr}+\mathrm{Cho})$ reductions providing lateralizing information. This might also explain why four patients (Pat No 4, 5, 9, 14) had only contralateral abnormalities. Future studies should combine 3D EPSI with a single slice hippocampal measurement to ensure that data is also consistently collected from these regions. (2) For the reasons outlined in the methodological sections, we used $\mathrm{NAA} /(\mathrm{Cr}+\mathrm{Cho})$ maps and not single metabolite maps. Therefore, we cannot exclude that some of the metabolic abnormalities were caused by elevated Cho and/or $\mathrm{Cr}$ concentrations rather than by NAA decreases. (3) The study population was small and so lack of statistical power might be responsible for some of the negative findings, e.g., such as not finding significant ipsi-/ contralateral differences. (4) All patients were recruited from tertiary care centers where they were evaluated for epilepsy surgery. These patients were probably suffering from a more severe form of TLE and, thus, may not be representative for non-lesional TLE in general. It will therefore be necessary to replicate the findings in a larger, more diverse TLE population. In addition to duration of epilepsy, seizure frequency, etc., future studies should also address the influence of other factors on extrafocal NAA/ $(\mathrm{Cr}+\mathrm{Cho})$ reductions, e.g., contribution of antiepileptic treatment, correlation with extent of EEG abnormalities.

\section{Conclusion}

This study confirmed the findings of previous spectroscopic imaging studies which demonstrated wide spread extrafocal NAA/(Cr+Cho) abnormalities in TLE-MTS and TLEno. The finding that extrafocal $\mathrm{NAA} /(\mathrm{Cr}+\mathrm{Cho})$ reductions show a considerable individual variation in extent and severity within each of the subtypes demonstrates that a homogeneous pattern of neuronal dysfunction/neuronal loss does not exist in either TLE-MTS or TLE-no. Further studies will be necessary to obtain a better understanding of the nature of these metabolic abnormalities and of their influence on cognitive performance and long term outcome after epilepsy surgery.

Acknowledgments This work was supported by the National Institute of Health grant RO1-NS31966 to K.D.L. None of the authors has any conflict of interest to disclose.

Open Access This article is distributed under the terms of the Creative Commons Attribution Noncommercial License which permits any noncommercial use, distribution, and reproduction in any medium, provided the original author(s) and source are credited.

\section{References}

1. Carne RP, Cook MJ, MacGregor LR, Kilpatrick CJ, Hicks RJ, O'Brien TJ (2007) Magnetic resonance imaging negative positron emission tomography positive temporal lobe epilepsy: FDG-PET pattern differs from mesial temporal lobe epilepsy. Mol Imaging Biol 9:32-42

2. Mueller SG, Laxer KD, Schuff N, Weiner MW (2007) Voxelbased $\mathrm{T} 2$ relaxation rate measurements in temporal lobe epilepsy (TLE) with and without mesial temporal sclerosis. Epilepsia 48:220-280

3. Bernasconi N, Bernasconi A, Caramanos Z, Dubeau F, Richardson J, Andermann F, Arnold DL (2001) Entorhinal cortex atrophy in epilepsy patients exhibiting normal hippocampal volumes. Neurology 56:1335-1339

4. Keller SS, Roberts N (2008) Voxel-based morphometry of temporal lobe epilepsy : an introduction and review of the literature. Epilepsia 49:741-757

5. Bernhardt BC, Worsley KJ, Besson P, Concha L, Lerch JL, Evans AC, Bernasconi N (2008) Mapping limbic network organization in temporal lobe epilepsy using morphometric correlations insights on the relation between mesiotemporal connectivity and cortical atrophy. Neuroimage 42:515-524

6. Vossler DG, Kramer DL, Haltiner AM, Rostad SW, Kjos BO, Davis BJ, Moran JD, Caylor LM (2004) Intracranial EEG in temporal lobe epilepsy: location of seizure onset relates to degree of hippocampal pathology. Epilepsia 45:450-497

7. Cohen-Gadol AA, Bradley CC, Williamson A, Kim JH, Westerveld M, Duckrow RB, Spencer DD (2005) Normal magnetic resonance imaging and medial temporal lobe epilepsy: the clinical syndrome of paradoxical temporal lobe epilepsy. J Neurosurg 102:902-909

8. Bell ML, Rao S, So EL, Trenerry M, Kazemi N, Stead SM, Cascino G, Marsh R, Meyer B, Watson RE, Giannini C, Worrell GA (2009) Epilepsy surgery outcome in temporal lobe epilepsy with a normal MRI. Epilepsia 50:2053-2060

9. Cohen-Gadol AA, Pan JW, Kim JH, Spencer DD, Hetherington HH (2004) Mesial temporal lobe epilepsy: a proton magnetic resonance spectroscopy study and histopathological analysis. J Neurosurg 101:613-620

10. Hammen T, Hildebrandt M, Stadlbauer A, Engelhorn T, Doelken M, Kerling F, Kaspar B, Romstoeck J, Ganslandt O, Nimsky C, Blumcke I, Doerfler A, Stefan H (2008) Non-invasive detection of hippocampal sclerosis: correlation between metabolite alterations detected by (1)H-MRS and neuropathology. NMR Biomed 21:545-552

11. Vermathen P, Laxer KD, Matson GB, Weiner MW (2000) Hippocampal stuctures: anteroposterior $N$-acetylaspartate differences in patients with epilepsy and control subjects as shown with proton MR spectroscopic imaging. Radiology 214:403-410 
12. Capizzano AA, Vermathen P, Laxer KD, Matson GB, Maudsley AA, Soher BJ, Schuff N, Weiner MW (2002) Multisection proton MR spectroscopy for mesial temporal lobe epilepsy. Am J Neuroradiol 23:1359-1368

13. Mueller SG, Laxer KD, Cashdollar N, Flenniken DL, Matson GB, Weiner MW (2004) Identification of abnormal neuronal metabolism outside the seizure focus in temporal lobe epilepsy. Epilepsia 45:355-366

14. Maudsley AA, Domenig C, Ramsay RE, Bowen BC (2010) Application of volumetric MR spectroscopic imaging for localization of neocortical epilepsy. Epilepsy Res 88:127-138

15. Ebel A, Maudsley AA (2003) Improved spectral quality for 3D MR spectroscopic imaging using a high spatial resolution acquisition strategy. Mag Reson Imaging 21:113-120

16. Mueller SG, Laxer KD, Barakos J, Cheong I, Garcia P, Weiner MW (2009) Widespread neocortical abnormalities in temporal lobe epilepsy with and without mesial temporal sclerosis. Neuroimage 46:353-359

17. Mueller SG, Laxer KD, Barakos J, Cheong I, Garcia P, Weiner MW (2009) Subfield atrophy pattern in temporal lobe epilepsy with and without mesial sclerosis detected by high resolution MRI at 4 Tesla: preliminary results. Epilepsia 50:1474-1483

18. Ebel A, Maudsely AA, Schuff N (2007) Correction of local B0 shifts in 3D EPSI of the human brain at 4T. Magn Reson Imaging 25:377-380

19. Soher BJ, Young K, Govindaraju V, Maudsley AA (1998) Automated spectral analysis III: application to in vivo proton spectroscopy and spectroscopic imaging. Magn Reson Med 40:822-831

20. Ebel A, Soher BJ, Maudsely AA (2001) Assessment of 3D proton MR echo-planar spectroscopic imaging using automated spectral analysis. Mag Reson Med 46:1072-1078
21. Van Leemput K, Maes F, Vandermeulen D, Suetens P (1999) Automated model-based bias field correction of MR images of the brain. IEEE Trans Med Imaging 10:885-896

22. Van Leemput K, Maes F, Vandermeulen D, Suetens P (1999) Automated model-based tissue classification of MR images of the brain. IEEE Trans Med Imaging 18:897-908

23. Studholme C, Hill DL, Hawkes DJ (1996) Automated 3-D registration of MR and CT images of the head. Med Image Anal $1: 163-175$

24. Connelly A, Jackson GD, Duncan JS, King MD, Gadian DG (1994) Magnetic resonance spectroscopy in temporal lobe epilepsy. Neurology 44:1411-1417

25. Simister RJ, McLean MA, Barker GJ, Duncan JS (2009) Proton MR spectroscopy of metabolite concentrations in temporal lobe epilepsy and effect of temporal lobe resection. Epilepsy Res 83:168-176

26. Serles W, Li LM, Antel SB, Cendes F, Gotman J, Olivier A, Andermann F, Dubeau F, Arnold DL (2001) Time course of postoperative recovery of $\mathrm{N}$-actyl-aspartate in temporal lobe epilepsy. Epilepsia 42:190-197

27. McDonald CR, Hagler D, Ahmadi ME, Tecoma E, Iragui V, Gharapetian L, Dale AM, Halgren E (2008) Regional neocortical thinning in mesial temporal lobe epilepsy. Epilepsia 49:794-803

28. Coan A, Appenzeller S, Bonilha L, Li LM, Cendes F (2009) Seizure frequency and lateralization affect progression of atrophy intemporal lobe epilepsy. Neurology 73:834-842

29. Simister RJ, McLean MA, Salmenpera TM, Barjer GJ, Duncan JS (2008) The effect of epileptic seizures on proton MRS visible neurochemical concentrations. Epilepsy Res 81:36-43

30. Savic I, Altshuler L, Baxter L, Engel J (1997) Pattern of interictal hypometabolism in PET scans with fludeoxyglucose F18 reflects prior seizure types in patients with mesial temporal lobe seizures. Arch Neurol 54:129-136 